\title{
System Usability Scale (SUS) on local language: A Sri Lankan case study
}

\author{
G.G.A.K. Kulatunga ${ }^{1 *}$, P.E.K.B. Ranatunga ${ }^{1}$, J.J. Hariyaram ${ }^{2}$, K. Kasturiaratchi ${ }^{2}$ \\ 1 Faculty of Medicine, University of Colombo, Colombo 08, Sri Lanka \\ 2Family Health Bureau, Colombo 10, Sri Lanka \\ *gumindu007@gmail.com \\ https://orcid.org/0000-0003-2164-5361
}

\begin{abstract}
Introduction: There are many information systems in Sri Lanka. However, there is no widely accepted tool to measure their usability. System Usability Scale (SUS) is a standardised metric or a quantitative measuring scale for measuring the usability of a website, information system or interactive system. SUS was released to the world by John Brooke in 1986. Currently, SUS has become an industry standard. In SUS, a user responds to 10 short questions on a 5-point Likert scale about the level of agreement. A special methodology is used to prevent unintentional rating responses. A SUS score above 68 would be considered above average. This questionnaire is originally in English language. However, even a native English speaker has implications on reliability and validity of the SUS due to the misunderstanding and misinterpretation of terminology or conflicting language. Thus, translating to native local languages is considered to increase the reliability and validity of SUS. The aim was to develop a Sinhala/Tamil System Usability Scale (SUS) to be used in Sri Lanka for assessing the information system usability.
\end{abstract}

Methods: Translation of SUS questions in English to Sinhala and Tamil Languages after brainstorming sessions. Questions were validated by multi-disciplinary specialists. Translations were aimed at keeping the content simple, clear and concise, achieving a conceptual translation rather than a literal translation and avoid technology jargon. The tool was pretested by using a group of users in Electronic Reproductive Health Information Management System (eRHMIS) of the Family Health Bureau.

Results: Sinhala/Tamil questions were finalized, and final SUS questionnaire was developed including a 5-point Likert scale. This tool was selected for measuring the usability of the eRHMIS of Family Health Bureau.

Conclusions: There was immediate acceptance as well as plans for future use of translated SUS. This SUS questionnaire can be used to measure the usability of existing information systems in the country.

Keywords: System Usability Scale, SUS, Information systems, Questionnaire, erhmis 